\title{
Development and validation of GMI signature based random survival forest prognosis model to predict clinical outcome in acute myeloid leukemia
}

Mingguang Shi ${ }^{*}$ and Guofu Xu

\begin{abstract}
Background: Acute myeloid leukemia (AML) is a disease with marked molecular heterogeneity and a high early death rate. Our aim was to investigate an integrated Gene expression, Mirna and miRNA-mRNA Interactions (GMI) signature for improving risk stratification of AML.

Methods: We identified differentially expressed genes by pooling a large number of 861 human AML patients and 75 normal cases. We then used miRWalk to identify the functional miRNA-mRNA regulatory module. The GMI signature based random survival forest (RSF) prognosis model was developed from training data set and evaluated in independent patient cohorts from The Cancer Genome Atlas (TCGA) dataset ( $N=147)$. Univariate and multivariate Cox proportional hazards regression analyses were applied to evaluate the prognostic value of GMI signature.

Results: We identified 139 differentially expressed genes between normal and abnormal AML samples. We discovered the functional miRNA-mRNA regulatory module which participate in the network of cancer progression. We named 23 differentially expressed genes and 16 validated target miRNAs as the GMI signature. The RSF model-based scores separated independent patient cohorts into two groups with significantly different overall survival (C-index $=0.59$, hazard ratio $[\mathrm{HR}], 2.12 ; 95 \%$ confidence interval $[\mathrm{Cl}], 1.11-4.03 ; p=0.019)$. Similar results were obtained with reversed training and testing datasets ( $C$-index $=0.58$, hazard ratio $[\mathrm{HR}], 2.08 ; 95 \%$ confidence interval $[\mathrm{Cl}], 1.02-4.24 ; p=0.038$ ). The GMl signature score contributed more information about recurrence than standard clinical covariates.

Conclusion: The GMI signature based RSF prognosis model not only reflects regulatory relationships from identified miRNA-mRNA module but also informs patient prognosis. While in the TCGA data set the GMI signature score contributed additional information about recurrence in comparison to standard clinical covariates, further studies are needed to determine its clinical significance.
\end{abstract}

Keywords: Acute myeloid leukemia, A GMI signature, The functional miRNA-mRNA regulatory module, Random survival forest

\footnotetext{
* Correspondence: mingguang.shi@hfut.edu.cn

School of Electric Engineering and Automation, Hefei University of

Technology, Hefei 230009, Anhui, China
}

(c) The Author(s). 2019 Open Access This article is distributed under the terms of the Creative Commons Attribution 4.0 International License (http://creativecommons.org/licenses/by/4.0/), which permits unrestricted use, distribution, and reproduction in any medium, provided you give appropriate credit to the original author(s) and the source, provide a link to the Creative Commons license, and indicate if changes were made. The Creative Commons Public Domain Dedication waiver (http://creativecommons.org/publicdomain/zero/1.0/) applies to the data made available in this article, unless otherwise stated. 


\section{Background}

Acute myeloid leukemia (AML) is a malignant disease of the bone marrow and typically represents functionally and phenotypically various cells in the same patient. Gene mutations identified distinct cytogenetically defined subsets of AML and unraveled the heterogeneous disorder in terms of genetic basis. Karyotype [1], mutations in the transcription factor CCAAT/enhancer-binding protein alpha $(C E B P A)[2,3]$, internal tandem duplications of the fms-related tyrosine kinase 3 (FLT3-ITD) [4], recurrent lesions in the nucleophosmin gene (NPM1) [5], GATA binding protein 2(GATA2) mutations [6], and Wilms tumor 1 (WT1) mutations [7] are related with patient relapse, prognosis and survival outcome. Although these guidelines for clinical treatment have improved the prognosis, AML is curable in about $35 \%$ of patients under 60 years old and $10 \%$ over 60 years old [8]. Hence, it is crucial to develop a reliable method for identifying new biomarkers and developing prognosis model to guide individual treatment of patients.

Several methods have been developed for the analysis of multiple molecular data to identify cancer-driven signatures and predict clinical outcome. Previous studies have demonstrated that microRNA signatures were identified to be associated with cytogenetics, prognosis and therapeutic targets in AML [9-11] and microRNA expressionbased model could predict event-free survival in AML [12, 13]. Moreover, distinct molecular subgroups that reflect discrete paths in the evolution of AML was identified to inform disease classification and prognostic stratification [14]. High-throughput proteomics data, such as Reverse Phase Proteomic Arrays (RPPA), was utilized to develop the prognosis model and bridge the gap between the underlying genetic alterations and functional cellular changes [15]. With the advances in next-generation sequencing (NGS) studies, integration of multiple molecular data and genomic knowledge improved the understanding of molecular pathogenesis and underlying biology in cancer [16]. Integrative data analysis methods led to the identification of novel microRNA-target gene interactions of potential relevance [17] and the discovery of NPM1 mutation-modulated miRNA-mRNA regulation pairs [18] for AML treatment. However, the sample size is still relatively small and the prognosis model has not been mentioned for patient stratification.

To address these questions, we collected 25 publicly available gene expression data sets containing 861 human AML patients and 75 normal cases. By pooling such a large amount of data, we aimed to identify differentially expressed genes for describing different gene expression patterns between normal samples and AML samples. Furthermore, we wanted to discover the functional regulatory networks for identifying potential regulations between mRNA and miRNA in biological processes. Based on the observation that miRNA-mRNA interactions were biologically relevant, we hypothesized that a functional miRNA-mRNA regulatory module could better represent underlying biological characteristics and may lead to a more powerful prognosis model. Moreover, we tried to develop Random survival forest (RSF) model using The Cancer Genome Atlas (TCGA) dataset based on prognostic miRNA/mRNA signatures. The results demonstrated that the model-based patient stratification provided useful predictive information for AML patient subgroups.

\section{Methods \\ Study design}

Figure 1 illustrated the overview of the overall study design. Microarray gene expression data on AML were collected and processed. Differential gene expression analysis was performed to identify differentially expressed genes. MiRWalk was then used to discover miRNAs that target the prioritized genes and further identify the functional miRNA-mRNA regulatory module. Specifically, the list of differentially expressed 23 genes and 16 target-validated miRNAs were named as the GMI signature because it integrated information from Gene expression, Mirna and miRNA-mRNA Interactions. Random survival forest (RSF) method were used with GMI signature to develop prognosis model in training cohort and then evaluated on the test dataset. We used RNA-seq and miRNA expression dataset on AML cases from TCGA as training and validation cohort. The gained RSF-based score was applied for patient stratification and survival analysis.

\section{Sample collection of AML datasets}

We searched PubMed (https://www.ncbi.nlm.nih.gov/ pubmed) with the terms "acute myeloid leukemia", "gene expression", "prognosis" and "signature" for published articles. This search retrieved the relevant Gene Expression Omnibus (GEO) database for further analysis. Raw microarray data were obtained from publicly available AML gene expression datasets in NCBI GEO. We collected AML samples of 25 gene expression datasets with Affymetrix $\mathrm{Hu}$ man Genome U133 plus 2.0 microarrays. The normalization was conducted on all samples in a single set using Robust MultiChip Analysis (RMA) algorithm [19] and Quantile Normalization and thus ensured the independence of processed datasets. Signal intensities were $\log 2$ transformed and probe set values summarized using Median Polish Summarization Method [20]. Probe set identifiers (IDs) were mapped to gene symbols based on the mapping from the GEO database. To resolve redundancies, multiple probes were mapped to unique Entrez Gene IDs by their median expression level. To make expression level comparable across genes, expression values for each gene were standardized using a Z-score transformation. An experimental group including 861 AML samples and a 


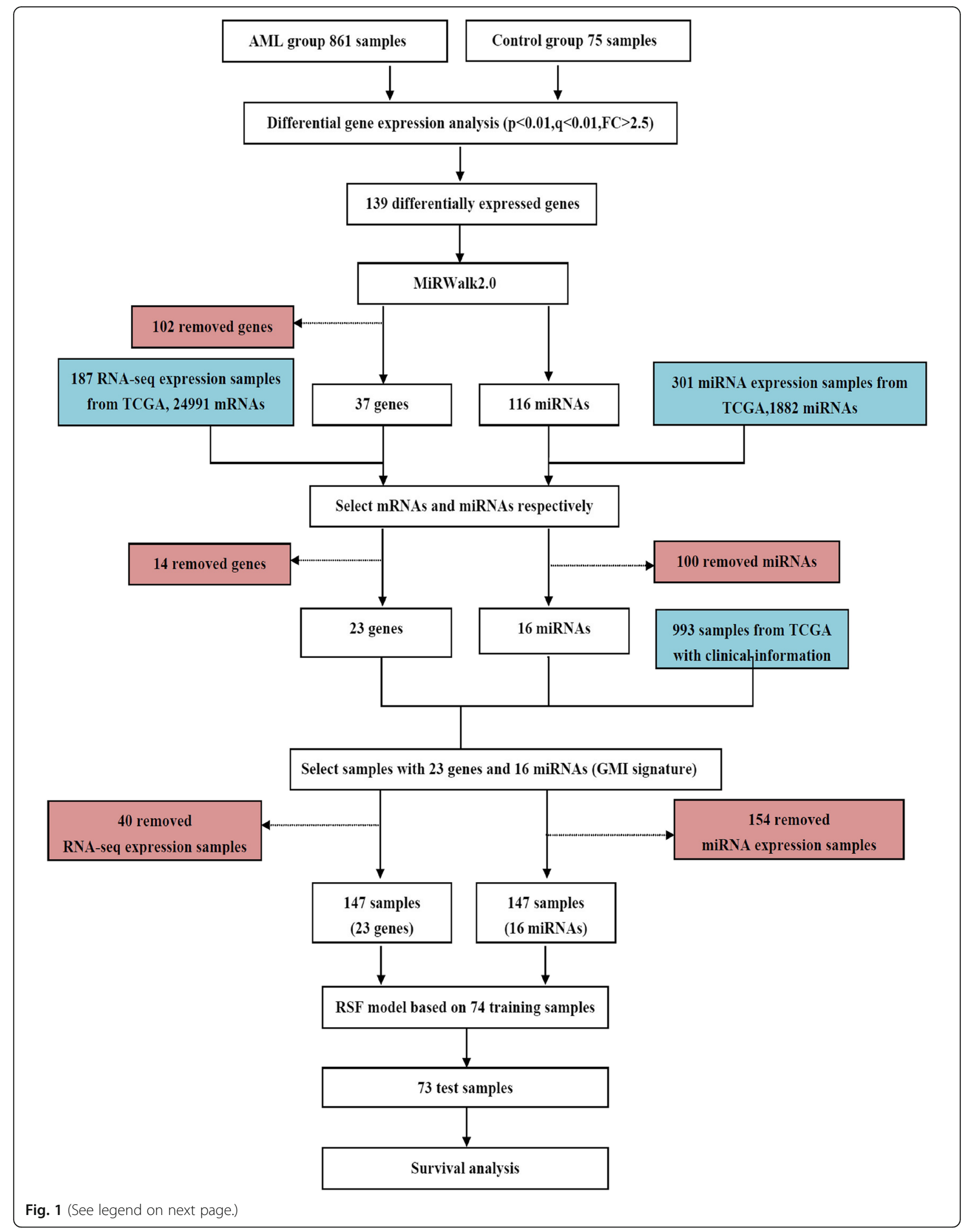


(See figure on previous page.)

Fig. 1 Outline of the study design. Differentially expressed genes were identified with differential expression analysis. MiRWalk was used to reconstruct the functional miRNA-mRNA regulatory module. RSF method was then used with GMI signature to develop prognosis model in training cohort. The developed prognosis model was evaluated on the independent dataset. The gained RSF-based score was applied for survival analysis and patient stratification. Specifically, we removed 14 genes which were not found in 187 RNA-seq expression dataset and 100 miRNAs which were not found in 301 miRNA expression dataset. Samples from TCGA with no expression of the signatures genes/miRNAs were filtered out. One hundred forty-seven AML samples were selected based on the present expression values of 23 gene expression signatures from 187 RNA-seq expression dataset and 16 miRNAs from 301 miRNA expression dataset

control group containing 75 normal bone marrow samples were used for the prioritization of gene expression signatures. The information of the source datasets of all AML samples is shown in the Table 1.

\section{TCGA samples for training and validation cohorts}

Transcriptomic data from the AML cohort were downloaded from the TCGA site (https://portal.gdc.cancer. gov/). We collected the mRNA sample data $(N=187,24$, 991 genes), the miRNA sample data $(N=301,1882$ miRNA) and the clinical sample data $(N=993)$ of AML from TCGA database. Log transformation (base 2) was used to re-scale mRNA and miRNA expression, followed by a Z-score transformation. Transcriptomic data of GMI signature were obtained and processed from TCGA. Specifically, 147 AML samples were selected based on the present expression values of 23 gene expression signatures from 187 RNA-seq expression dataset and 16 miRNAs from 301 miRNA expression dataset.

Data collection and process was shown in Fig. 1. We split the tissue samples into TCGA Part One (TCGAPO, $N=74$ ) and TCGA Part Two (TCGAPT, $N=73$ ) cohorts for further analysis. TCGAPO cohort includes 41 samples (alive) and 33 samples (dead) for overall survival (OS) of patients. At the same time, TCGAPT cohort contains 33 samples (alive) and 40 samples (dead) for OS of cancer patients. Each dataset was used as a training-set in turn and developed models were evaluated against the other dataset. Table 2 illustrated the clinical characteristics of TCGA AML cohort.

\section{Differential gene expression analysis}

To identify differentially expressed genes, differential expression analysis was used to assess differences in gene expression between an experimental group and a control group assessed by two-tailed Student's $t$-tests and corrected by Benjamini-Hochberg [44]. The false discovery rate (FDR) of multiple testing was controlled using the Benjamini and Hochberg method. Significantly differentially expressed genes were selected with the FDRadjusted $p$-values $<0.01$. Adjusted statistical significance was then set at q-values $<0.01$ with FDR correction for multiple testing where relevant. Fold Change value of 2.5 was further used as a cut-off to identify up- and downregulated genes.

\section{MiRWalk identify miRNA-mRNA interactions}

To identify miRNA-mRNA interactions, we used the significantly differentially expressed genes (mRNAs) as seeds and identified the target-validated miRNAs from the miRWalk2.0 database (http://mirwalk.umm.uni-heidelberg.de/) [45]. To investigate the biological relevance of the identified interactions, we used an advanced search options including miRDB and TargetScan for miRNAs target selection. The functionally correlated miRNA-mRNA regulatory module was then identified and constructed for further analysis.

\section{Survival analysis for GMI signature}

The LinkedOmics database (http://www.linkedomics. org) contains multiple molecular data and clinical data for different cancer types from the TCGA project, which systematically interpret and explore the complex relationships between the vast amount of clinical and molecular attributes [46]. In addition, Gene Expression Profiling Interactive Analysis (GEPIA) (http://gepia.cancer-pku.cn/) was used for efficiently analyzing the RNA sequencing expression data from the TCGA data [47]. In this analysis, we utilized these two analytical tools to perform validation of AML specific expression and prognosis for the GMI signature.

\section{Statistical analysis}

We used random survival forest (RSF) method for developing a prognosis model [48]. An $\mathrm{R}$ implementation of the $r f s r c$ available in the randomForestSRC package was used for model development. RSF had two parameters ntree and mtry, where ntree represented the number of trees in the forest and mtry was the number of randomly selected variables for splitting at each node. We used a grid-search on ntree and mtry using 5-fold crossvalidation. All the pairs of (ntree, mtry) are formed and the one with the best $\mathrm{C}$-index value is identified as the optimized parameters. The $\mathrm{C}$-index represents a probability of the concordance between predicted and observed survival, which is a typical metric for quantifying the predictive ability of a survival model. The developed RSF prognosis model based on the optimal parameters was then evaluated on the independent dataset where the RSF-based score was derived for each sample. 
Table 1 AML gene expression datasets used to prioritize the gene expression signatures

\begin{tabular}{|c|c|c|c|c|c|c|c|}
\hline Data Set & Country & $\begin{array}{l}\text { Control } \\
\text { group }\end{array}$ & $\begin{array}{l}\text { Experimental } \\
\text { Group }\end{array}$ & Author & Title & Journal & Reference \\
\hline GSE14924 & $\begin{array}{l}\text { United } \\
\text { Kingdom }\end{array}$ & 21 & 20 & (Le et al. 2009) & $\begin{array}{l}\text { Peripheral blood T cells in acute myeloid } \\
\text { leukemia (AML) patients at diagnosis have } \\
\text { abnormal phenotype and genotype and } \\
\text { form defective immune synapses with AML } \\
\text { blasts }\end{array}$ & Blood & [21] \\
\hline GSE68172 & Germany & 5 & 72 & $\begin{array}{l}\text { (Schneider et al. } \\
\text { 2015) }\end{array}$ & $\begin{array}{l}\text { Leukemic progenitor cells are susceptible } \\
\text { to targeting by stimulated cytotoxic T cells } \\
\text { against immunogenic leukemia-associated } \\
\text { antigens }\end{array}$ & $\begin{array}{l}\text { International Journal of } \\
\text { Cancer }\end{array}$ & [22] \\
\hline GSE84881 & Germany & 4 & 19 & (Ek et al. 2016) & $\begin{array}{l}\text { Molecular alterations in bone marrow } \\
\text { mesenchymal stromal cells derived from } \\
\text { acute myeloid leukemia patients }\end{array}$ & Leukemia & [23] \\
\hline GSE14858 & Italy & 20 & 20 & $\begin{array}{l}\text { (Bresolin et al. } \\
\text { 2010) }\end{array}$ & $\begin{array}{l}\text { Gene expression-based classification as an } \\
\text { independent predictor of clinical outcome } \\
\text { in juvenile myelomonocytic leukemia }\end{array}$ & $\begin{array}{l}\text { Journal of Clinical } \\
\text { Oncology }\end{array}$ & [24] \\
\hline GSE12662 & USA & 15 & 91 & $\begin{array}{l}\text { (Payton et al. } \\
\text { 2009) }\end{array}$ & $\begin{array}{l}\text { High throughput digital quantification of } \\
\text { mRNA abundance in primary human acute } \\
\text { myeloid leukemia samples }\end{array}$ & $\begin{array}{l}\text { Journal of Clinical } \\
\text { Investigation }\end{array}$ & [25] \\
\hline GSE10746 & USA & 3 & 8 & $\begin{array}{l}\text { (Mougeot et al. } \\
\text { 2011) }\end{array}$ & $\begin{array}{l}\text { Microarray analyses of oral punch biopsies } \\
\text { from acute myeloid leukemia (AML) } \\
\text { patients treated with chemotherapy }\end{array}$ & $\begin{array}{l}\text { Oral Surgery Oral } \\
\text { Medicine Oral } \\
\text { Pathology Oral } \\
\text { Radiology \& } \\
\text { Endodontology }\end{array}$ & [26] \\
\hline GSE17054 & USA & 4 & 9 & $\begin{array}{l}\text { (Majeti et al. } \\
\text { 2009) }\end{array}$ & $\begin{array}{l}\text { Dysregulated gene expression networks in } \\
\text { human acute myelogenous leukemia stem } \\
\text { cells }\end{array}$ & $\begin{array}{l}\text { Proceedings of the } \\
\text { National Academy of } \\
\text { Sciences of the United } \\
\text { States of America }\end{array}$ & [27] \\
\hline GSE8023 & USA & 3 & 9 & $\begin{array}{l}\text { (Krejci et al. } \\
2008)\end{array}$ & $\begin{array}{l}\text { p53 signaling in response to increased } \\
\text { DNA damage sensitizes AML1-ETO cells to } \\
\text { stress-induced death }\end{array}$ & Blood & [28] \\
\hline GSE17061 & Netherlands & 0 & 35 & $\begin{array}{l}\text { (Silva et al. } \\
\text { 2009) }\end{array}$ & $\begin{array}{l}\text { Gene expression profiling of minimally } \\
\text { differentiated acute myeloid leukemia: MO } \\
\text { is a distinct entity subdivided by RUNX1 } \\
\text { mutation status }\end{array}$ & Blood & [29] \\
\hline GSE70124 & Germany & 0 & 46 & $\begin{array}{l}\text { (Papaemmanuil } \\
\text { et al. 2016) }\end{array}$ & $\begin{array}{l}\text { Genomic Classification and Prognosis in } \\
\text { Acute Myeloid Leukemia }\end{array}$ & $\begin{array}{l}\text { New England Journal } \\
\text { of Medicine }\end{array}$ & [14] \\
\hline GSE10258 & Austria & 0 & 15 & $\begin{array}{l}\text { (Zatkova et al. } \\
\text { 2009) }\end{array}$ & $\begin{array}{l}\text { AML/MDS with } 11 \mathrm{q} / \mathrm{MLL} \text { amplification } \\
\text { show characteristic gene expression } \\
\text { signature and interplay of DNA copy } \\
\text { number changes }\end{array}$ & $\begin{array}{l}\text { Genes Chromosomes \& } \\
\text { Cancer }\end{array}$ & [30] \\
\hline GSE35159 & Japan & 0 & 12 & $\begin{array}{l}\text { (Saito et al. } \\
\text { 2011) }\end{array}$ & $\begin{array}{l}\text { CD52 as a molecular target for } \\
\text { immunotherapy to treat acute myeloid } \\
\text { leukemia with high EVI1 expression }\end{array}$ & Leukemia & [31] \\
\hline GSE50928 & France & 0 & 13 & $\begin{array}{l}\text { (Khaznadar et } \\
\text { al. 2015) }\end{array}$ & $\begin{array}{l}\text { Defective NK Cells in Acute Myeloid } \\
\text { Leukemia Patients at Diagnosis Are } \\
\text { Associated with Blast Transcriptional } \\
\text { Signatures of Immune Evasion }\end{array}$ & Journal of Immunology & [32] \\
\hline GSE34885 & France & 0 & 14 & $\begin{array}{l}\text { (Khaznadar et } \\
\text { al. 2015) }\end{array}$ & $\begin{array}{l}\text { Defective NK Cells in Acute Myeloid } \\
\text { Leukemia Patients at Diagnosis Are } \\
\text { Associated with Blast Transcriptional } \\
\text { Signatures of Immune Evasion }\end{array}$ & Journal of Immunology & [32] \\
\hline GSE52891 & Netherlands & 0 & 23 & $\begin{array}{l}\text { (Bachas et al. } \\
\text { 2015) }\end{array}$ & $\begin{array}{l}\text { Gene Expression Profiles Associated with } \\
\text { Pediatric Relapsed AML }\end{array}$ & Plos One & [33] \\
\hline GSE22056 & Netherlands & 0 & 98 & $\begin{array}{l}\text { (de Jonge et al. } \\
\text { 2010) }\end{array}$ & $\begin{array}{l}\text { High VEGFC expression is associated with } \\
\text { unique gene expression profiles and } \\
\text { predicts adverse prognosis in pediatric and } \\
\text { adult acute myeloid leukemia }\end{array}$ & Blood & [34] \\
\hline GSE59808 & USA & 0 & 32 & (Guo et al. & PIM inhibitors target CD25-positive AML & Blood & [35] \\
\hline
\end{tabular}


Table 1 AML gene expression datasets used to prioritize the gene expression signatures (Continued)

\begin{tabular}{|c|c|c|c|c|c|c|c|}
\hline Data Set & Country & $\begin{array}{l}\text { Control } \\
\text { group }\end{array}$ & $\begin{array}{l}\text { Experimental } \\
\text { Group }\end{array}$ & Author & Title & Journal & Reference \\
\hline & & & & 2014) & $\begin{array}{l}\text { cells through concomitant suppression of } \\
\text { STAT5 activation and degradation of MYC } \\
\text { oncogene }\end{array}$ & & \\
\hline GSE12326 & China & 0 & 10 & $\begin{array}{l}\text { (Cheung et al. } \\
\text { 2009) }\end{array}$ & $\begin{array}{l}\text { A comparative study of bone marrow and } \\
\text { peripheral blood CD34+ myeloblasts in } \\
\text { acute myeloid leukaemia }\end{array}$ & $\begin{array}{l}\text { British Journal of } \\
\text { Haematology }\end{array}$ & [36] \\
\hline GSE44857 & $\begin{array}{l}\text { United } \\
\text { Kingdom }\end{array}$ & 0 & 18 & $\begin{array}{l}\text { (Leonard et al. } \\
\text { 2014) }\end{array}$ & $\begin{array}{l}\text { Sequential Treatment with Cytarabine and } \\
\text { Decitabine Has an Increased Anti-Leukemia } \\
\text { Effect Compared to Cytarabine Alone in } \\
\text { Xenograft Models of Childhood Acute } \\
\text { Myeloid Leukemia }\end{array}$ & Plos One & [37] \\
\hline GSE30903 & Italy & 0 & 24 & $\begin{array}{l}\text { (Salvestrini et al. } \\
\text { 2012) }\end{array}$ & $\begin{array}{l}\text { Purinergic signaling inhibits human acute } \\
\text { myeloblastic leukemia cell proliferation, } \\
\text { migration, and engraftment in } \\
\text { immunodeficient mice }\end{array}$ & Blood & [38] \\
\hline GSE22845 & Netherlands & 0 & 154 & $\begin{array}{l}\text { (Taskesen et al. } \\
\text { 2011) }\end{array}$ & $\begin{array}{l}\text { Prognostic impact, concurrent genetic } \\
\text { mutations, and gene expression features of } \\
\text { AML with CEBPA mutations in a cohort of } \\
1182 \text { cytogenetically normal AML patients: } \\
\text { further evidence for CEBPA double mutant } \\
\text { AML as a distinctive disease entity }\end{array}$ & Blood & [39] \\
\hline GSE18018 & USA & 0 & 19 & $\begin{array}{l}\text { (Falini et al. } \\
\text { 2010) }\end{array}$ & $\begin{array}{l}\text { Multilineage dysplasia has no impact on } \\
\text { biologic, clinicopathologic, and prognostic } \\
\text { features of AML with mutated } \\
\text { nucleophosmin (NPM1) }\end{array}$ & Blood & [40] \\
\hline GSE21261 & USA & 0 & 79 & $\begin{array}{l}\text { (Miesner et al. } \\
\text { 2010) }\end{array}$ & $\begin{array}{l}\text { Multilineage dysplasia (MLD) in acute } \\
\text { myeloid leukemia (AML) correlates with } \\
\text { MDS-related cytogenetic abnormalities and } \\
\text { a prior history of MDS or MDS/MPN but } \\
\text { has no independent prognostic relevance: } \\
\text { a comparison of } 408 \text { cases classified as } \\
\text { "AML not otherwise specified" (AML-NOS) } \\
\text { or "AML with myelodysplasia-related } \\
\text { changes" (AML-MRC) }\end{array}$ & Blood & [41] \\
\hline GSE56237 & Denmark & 0 & 10 & $\begin{array}{l}\text { (Mora-Jensen et } \\
\text { al. 2015) }\end{array}$ & $\begin{array}{l}\text { Cellular origin of prognostic chromosomal } \\
\text { aberrations in AML patients }\end{array}$ & Leukemia & [42] \\
\hline GSE30442 & USA & 0 & 11 & $\begin{array}{l}\text { (Grossmann et } \\
\text { al. 2011) }\end{array}$ & $\begin{array}{l}\text { Whole-exome sequencing identifies } \\
\text { somatic mutations of BCOR in acute } \\
\text { myeloid leukemia with normal karyotype }\end{array}$ & Blood & [43] \\
\hline
\end{tabular}

The calculated C-index values evaluated the association between the RSF-based score and real prognosis of the patients. Standard Kaplan-Meier survival curves were generated for different risk patient groups on the basis of the RSF-based scores. The median score was used to stratify patients into high-risk and low-risk score groups, and the log-rank test was utilized to assess the survival difference between two different risk groups. The statistical test was two-sided and the estimated $p$ value less than 0.05 was considered statistically significant.

\section{Results}

\section{The prioritized gene expression signatures}

In the microarray analysis, 139 genes were found to be differentially expressed between 75 normal bone marrow samples and 861 AML samples (Additional file 1: Table S1). All the 139 genes suggested higher expression levels with statistical significance in AML cases than in controls. We illustrated the volcano plot by analyzing genes with differential expressions between 861 AML samples and 75 normal cases for mRNA microarrays (Additional file 2: Figure S1). It showed the significant interactions with $-\log 10$ ( $p$-value) as a function of the $\log 2$ fold-change in the gene expression of AML.

\section{The functional miRNA-mRNA regulatory module}

MiRWalk2.0 is a publicly available comprehensive archive, providing an array of experimentally verified and predicted miRNA-mRNA interaction pairs. It has been proved that miRNA-mRNA interactions play critical roles in diverse biological processes and pathologies [49].

We used the above 139 differential expressed genes (mRNAs) to identify the correlated miRNAs which may target them. We then identified 37 mRNAs with matched 
Table 2 The clinical characteristics of AML patients from TCGA. CR, complete remission; MLL, mixed lineage leukemia

\begin{tabular}{|c|c|c|}
\hline & TCGAPO $(N=74)$ & TCGAPT $(N=73)$ \\
\hline \multicolumn{3}{|l|}{ Age at Diagnosis (year) } \\
\hline median (range) & $10.58(0.40-22.55)$ & $9.04(0.38-19.12)$ \\
\hline \multicolumn{3}{|l|}{ Gender } \\
\hline female (n\%) & $34(45.95 \%)$ & $40(54.79 \%)$ \\
\hline \multicolumn{3}{|l|}{ Race } \\
\hline white (n\%) & $59(79.73 \%)$ & $55(75.34 \%)$ \\
\hline \multicolumn{3}{|l|}{ First event } \\
\hline relapse (n\%) & $51(68.92 \%)$ & $54(73.97 \%)$ \\
\hline \multicolumn{3}{|l|}{ CR status } \\
\hline CR (n\%) & $69(93.24 \%)$ & $61(83.56 \%)$ \\
\hline \multicolumn{3}{|l|}{ Primary Cytogenetic } \\
\hline MLL (n\%) & $10(13.51 \%)$ & $18(24.66 \%)$ \\
\hline Normal (n\%) & $18(24.32 \%)$ & $11(15.07 \%)$ \\
\hline Other (n\%) & $14(18.92 \%)$ & $20(27.40 \%)$ \\
\hline$t(8 ; 21)$ & $7(9.46 \%)$ & $11(15.07 \%)$ \\
\hline inv.(16) & $16(21.62 \%)$ & $12(16.44 \%)$ \\
\hline \multicolumn{3}{|l|}{ Cytogenetic Site of Relapse/Induction Failure } \\
\hline Yes (n\%) & $4(5.41 \%)$ & $13(17.81 \%)$ \\
\hline No $(n \%)$ & $48(64.86 \%)$ & $43(58.90 \%)$ \\
\hline Not done (n\%) & $22(29.73 \%)$ & $17(23.29 \%)$ \\
\hline \multicolumn{3}{|l|}{ FAB Category } \\
\hline $\mathrm{M0}, \mathrm{M} 1, \mathrm{M} 2, \mathrm{M} 3, \mathrm{M} 4, \mathrm{M} 5, \mathrm{M} 6, \mathrm{M} 7, \mathrm{NOS}$ & $\begin{array}{l}2(2.70 \%), 7(9.46 \%), 19(25.68 \%), 0(0.00 \%), 22 \\
(29.73 \%), 15(20.27 \%), 1(1.35 \%), 2(2.70 \%), 5 \\
(6.76 \%)\end{array}$ & $\begin{array}{l}2(2.74 \%), 8(10.96 \%), 15(20.55 \%), 0(0.00 \%), 20 \\
(27.40 \%), 12(16.44 \%), 1(1.37 \%), 5(6.85 \%), 3 \\
(4.11 \%)\end{array}$ \\
\hline \multicolumn{3}{|l|}{ FLT3/ITD } \\
\hline Positive (n\%) & $8(10.81 \%)$ & $5(6.85 \%)$ \\
\hline Negative (n\%) & $66(89.19 \%)$ & $68(93.15 \%)$ \\
\hline \multicolumn{3}{|l|}{ WBC at Diagnosis } \\
\hline median (range) & $53.5(2.1-302)$ & $34.9(1.3-519)$ \\
\hline \multicolumn{3}{|l|}{ NPM mutation } \\
\hline Yes (n\%) & $4(5.41 \%)$ & $2(2.74 \%)$ \\
\hline No $(n \%)$ & $66(89.19 \%)$ & 70 (95.89\%) \\
\hline \multicolumn{3}{|l|}{ CEBPA mutation } \\
\hline Yes (n\%) & $4(5.41 \%)$ & $5(6.85 \%)$ \\
\hline No $(n \%)$ & $69(93.24 \%)$ & $67(91.78 \%)$ \\
\hline \multicolumn{3}{|l|}{ WT1 mutation } \\
\hline Yes (n\%) & $4(5.41 \%)$ & $6(8.22 \%)$ \\
\hline No (n\%) & $67(90.54 \%)$ & $66(90.41 \%)$ \\
\hline \multicolumn{3}{|l|}{ Protocol } \\
\hline CCG-2961(n\%), AAML03P1(n\%), AAML0531(n\%) & $18(24.3 \%), 38(51.4 \%), 18(24.3 \%)$ & $0(0 \%), 0(0 \%), 73(100 \%)$ \\
\hline
\end{tabular}

116 miRNAs (Additional file 2: Figure S2). One hundred two mRNAs were removed because these mRNAs have no matched miRNA targets. The functionally correlated miRNA-mRNA regulatory module (the center of Additional file 2: Figure S2) was then derived with 23 mRNAs and correlated 97 miRNAs (Fig. 2). Twenty-three differential expressed genes are then listed in Table 3. Among the 97 miRNAs, only the 16 miRNAs in the AML TCGA dataset were found and used for further analysis. A panel of 16 miRNA markers contained hsa-mir-448, hsa-mir- 
320a, hsa-mir-378b, hsa-mir-378c, hsa-mir-378f, hsa-mir378e, hsa-mir-378 h, hsa-mir-378i, hsa-mir-520b, hsa-mir520e, hsa-mir-429, hsa-mir-137, hsa-mir-1193, hsa-mir346, hsa-mir-449a, and hsa-mir-107. Specifically, we named the list of differentially expressed 23 genes and 16 target-validated miRNAs the GMI signature because it integrated information from Gene expression, Mirna and miRNA-mRNA Interactions.

Previous studies demonstrated that Sox4 [50], RasGRP1 [51], RasGRP3 [51], IGF1R [52], CDK6 [53], and LEF1 [54] are the key oncogenes/tumor suppressor genes in acute myeloid leukemia. Among the GMI signature, IGF1R is targeted by hsa-miR-378b, hsa-miR378c, hsa-miR-378e, hsa-miR-378f, hsa-miR-378 h, hsa- $m i R-378 i$, and $h s a-m i R-448$ respectively. It suggests that hsa-miR-378 family members and hsa-miR-448 have important regulatory functions for AML. In addition, AKTIP is targeted by $h s a-m i R-520 b$ and $h s a-m i R-520 e$, CDK6 is targeted by hsa-miR-320a and hsa-miR-449a, PAG1 is targeted by hsa-miR-429 and hsa-miR-1193, RORA is targeted by $h s a-m i R-107$ and $h s a-m i R-137$. It confirms that hsa-miR-520b, hsa-miR-520e, hsa-miR320a, hsa-miR-449a, hsa-miR-429, hsa-miR-1193, hsamiR-107, and hsa-miR-137 also play important roles in regulating AML. Moreover, LEF1 is targeted by $h s a-$ miR-449a, MGAT4A is targeted by hsa-miR-449a, PLXNC1 is targeted by hsa-miR-320a, and SCML4 is targeted by $h s a-m i R-346$. It indicates that $h s a-m i R-$

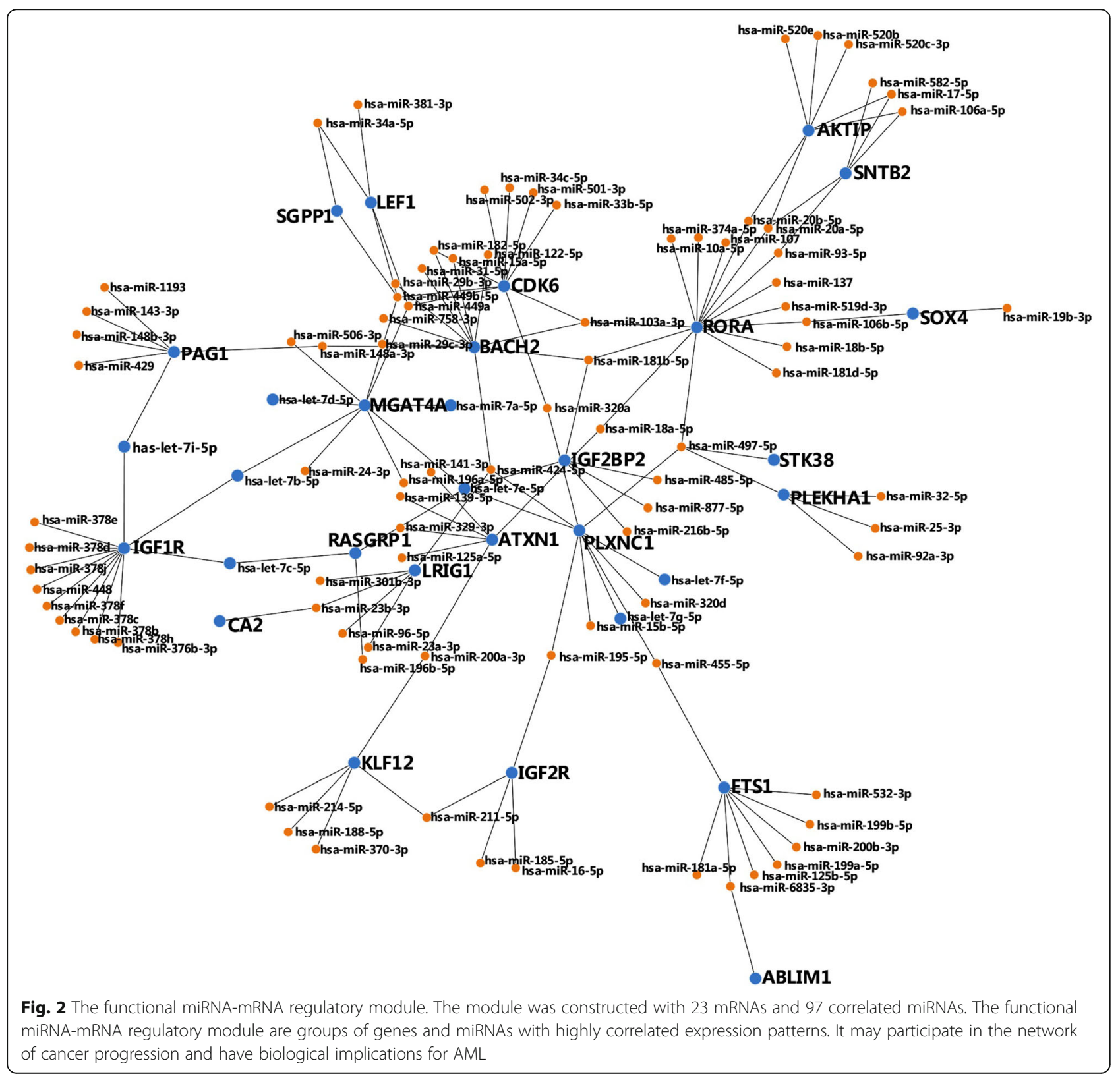


Table 3 The 23 differentially expressed genes

\begin{tabular}{|c|c|c|c|c|c|c|c|}
\hline $\begin{array}{l}\text { Gene } \\
\text { Symbol }\end{array}$ & Mean Signal of AML Group & $\begin{array}{l}\text { Mean Signal of Control } \\
\text { Group }\end{array}$ & t statistic & $\begin{array}{l}\text { Fold } \\
\text { Change }\end{array}$ & $p$-value & q-value & $\begin{array}{l}\text { Gene } \\
\text { Feature }\end{array}$ \\
\hline SOX4 & 9.112336 & 7.219739 & 7.675507 & 3.713032 & $\begin{array}{l}<1 \times 10- \\
6\end{array}$ & $\begin{array}{l}<1 \times 10- \\
6\end{array}$ & up \\
\hline RASGRP1 & 5.83589 & 7.652658 & -7.195507 & -3.522911 & $\begin{array}{l}<1 \times 10- \\
6\end{array}$ & $\begin{array}{l}<1 \times 10- \\
6\end{array}$ & down \\
\hline $\mathrm{BACH} 2$ & 4.320585 & 6.060818 & -8.152871 & -3.340891 & $\begin{array}{l}<1 \times 10- \\
6\end{array}$ & $\begin{array}{l}<1 \times 10- \\
6\end{array}$ & down \\
\hline KLF12 & 6.133251 & 7.861214 & -7.870257 & -3.312596 & $\begin{array}{l}<1 \times 10- \\
6\end{array}$ & $\begin{array}{l}<1 \times 10- \\
6\end{array}$ & down \\
\hline$L R / G 1$ & 4.728837 & 6.393224 & -9.836949 & -3.16979 & $\begin{array}{l}<1 \times 10- \\
6\end{array}$ & $\begin{array}{l}<1 \times 10- \\
6\end{array}$ & down \\
\hline ETS1 & 7.934244 & 9.498425 & -7.305467 & -2.957097 & $\begin{array}{l}<1 \times 10- \\
6\end{array}$ & $\begin{array}{l}<1 \times 10- \\
6\end{array}$ & down \\
\hline CA2 & 7.797592 & 6.234079 & 6.061318 & 2.955725 & $\begin{array}{l}<1 \times 10- \\
6\end{array}$ & $\begin{array}{l}<1 \times 10- \\
6\end{array}$ & up \\
\hline SGPP1 & 5.2489 & 6.809546 & -10.216815 & -2.949861 & $\begin{array}{l}<1 \times 10- \\
6\end{array}$ & $\begin{array}{l}<1 \times 10- \\
6\end{array}$ & down \\
\hline AKTIP & 5.928499 & 7.444547 & -11.312356 & -2.860065 & $\begin{array}{l}<1 \times 10- \\
6\end{array}$ & $\begin{array}{l}<1 \times 10- \\
6\end{array}$ & down \\
\hline MGAT4A & 6.147385 & 7.656076 & -6.959608 & -2.845517 & $\begin{array}{l}<1 \times 10- \\
6\end{array}$ & $\begin{array}{l}<1 \times 10- \\
6\end{array}$ & down \\
\hline IGF1R & 5.953049 & 7.435281 & -9.157838 & -2.793807 & $\begin{array}{l}<1 \times 10- \\
6\end{array}$ & $\begin{array}{l}<1 \times 10- \\
6\end{array}$ & down \\
\hline IGF2BP2 & 8.551588 & 7.080512 & 6.331802 & 2.772285 & $\begin{array}{l}<1 \times 10- \\
6\end{array}$ & $\begin{array}{l}<1 \times 10- \\
6\end{array}$ & up \\
\hline ATXN1 & 5.977417 & 7.443869 & -11.143182 & -2.763415 & $\begin{array}{l}<1 \times 10- \\
6\end{array}$ & $\begin{array}{l}<1 \times 10- \\
6\end{array}$ & down \\
\hline PLXNC1 & 6.920035 & 8.327643 & -7.413724 & -2.652969 & $\begin{array}{l}<1 \times 10- \\
6\end{array}$ & $\begin{array}{l}<1 \times 10- \\
6\end{array}$ & down \\
\hline PLEKHA1 & 5.191966 & 6.576699 & -6.150913 & -2.611237 & $\begin{array}{l}<1 \times 10- \\
6\end{array}$ & $\begin{array}{l}<1 \times 10- \\
6\end{array}$ & down \\
\hline PAG1 & 6.025085 & 7.409445 & -6.906294 & -2.61056 & $\begin{array}{l}<1 \times 10- \\
6\end{array}$ & $\begin{array}{l}<1 \times 10- \\
6\end{array}$ & down \\
\hline SNTB2 & 3.785427 & 5.155895 & -6.769114 & -2.585543 & $\begin{array}{l}<1 \times 10- \\
6\end{array}$ & $\begin{array}{l}<1 \times 10- \\
6\end{array}$ & down \\
\hline IGF2R & 6.569925 & 7.933466 & -6.300174 & -2.573159 & $\begin{array}{l}<1 \times 10- \\
6\end{array}$ & $\begin{array}{l}<1 \times 10- \\
6\end{array}$ & down \\
\hline STK38 & 4.463917 & 5.809704 & -6.984927 & -2.541687 & $\begin{array}{l}<1 \times 10- \\
6\end{array}$ & $\begin{array}{l}<1 \times 10- \\
6\end{array}$ & down \\
\hline CDK6 & 6.572063 & 5.241319 & 9.134775 & 2.515324 & $\begin{array}{l}<1 \times 10- \\
6\end{array}$ & $\begin{array}{l}<1 \times 10- \\
6\end{array}$ & up \\
\hline LEF1 & 6.517115 & 8.211854 & -5.602032 & -3.237184 & $\begin{array}{l}<1 \times 10- \\
6\end{array}$ & 0.000002 & down \\
\hline ABLIM1 & 7.040753 & 8.373263 & -5.375175 & -2.518404 & 0.000001 & 0.000004 & down \\
\hline RORA & 5.041835 & 6.718816 & -5.269869 & -3.197582 & 0.000001 & 0.000006 & down \\
\hline
\end{tabular}

449a, hsa-miR-320a, and hsa-miR-346 may be very significant for the regulation of AML.

Collectively, the identified interactions between miRNAs and mRNAs suggest that the functional miRNAmRNA regulatory module participate in the network of cancer progression and have biological implications for AML in common.
GMI signature in survival analysis

To analyze the predictive value of the GMI signature on survival we used the LinkedOmics tool. We got the survival analysis curves of each gene and target-validated miRNA from TCGA tumor samples (Fig. 3 and Additional file 2: Figure S3). Seven genes/miRNAs were found with statistical significance $(p \leq 0.05)$ including 


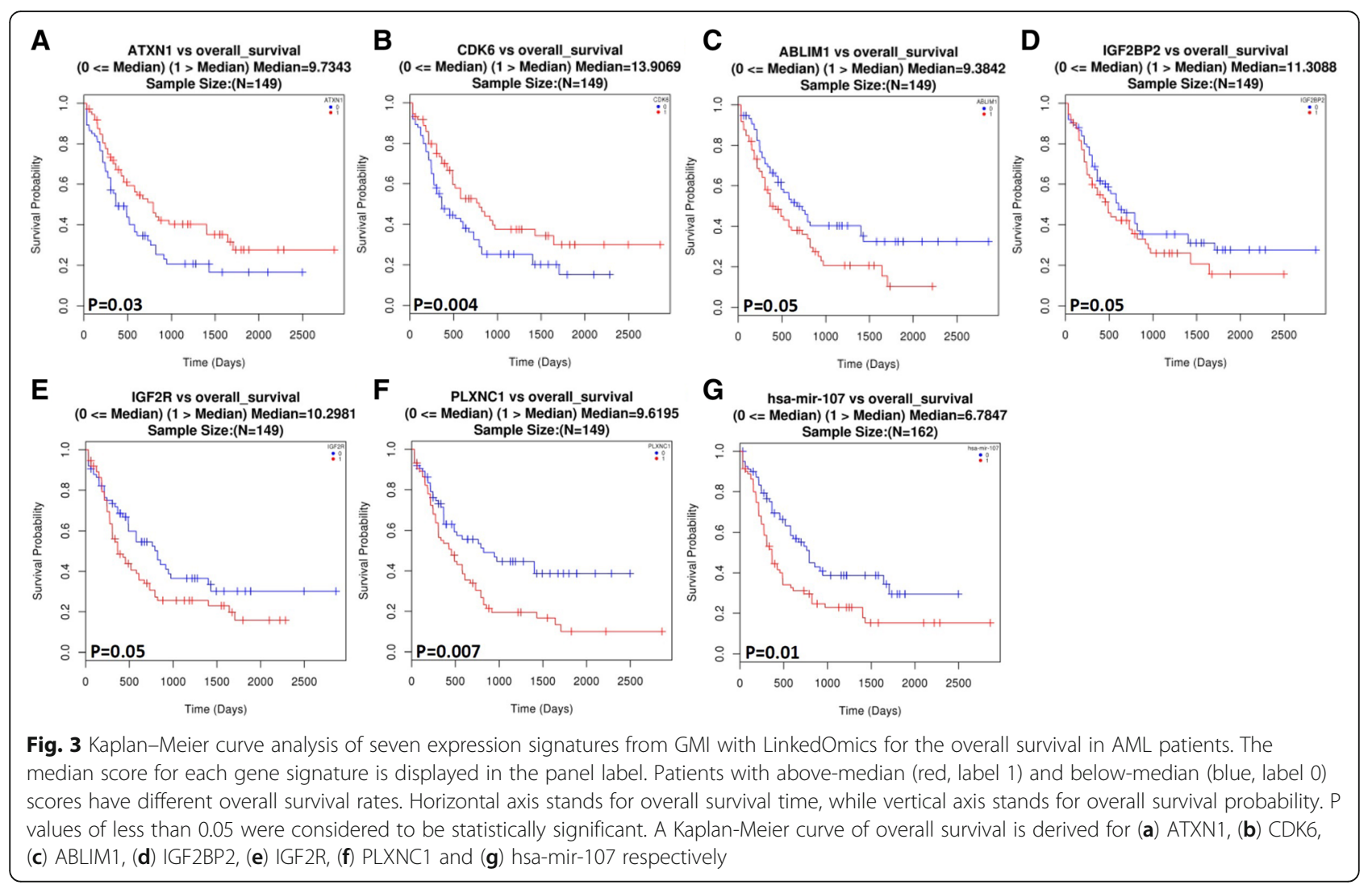

ABLIM1, ATXN1, CDK6, IGF2BP2, IGF2R, PLXNC1, and hsa-mir-107 (Fig. 3). It is worth noting that the high-risk group has significantly worse overall survival than the low-risk group for the previous genes/miRNAs.

Similarly, we applied GEPIA for the prognosis of the GMI signature in AML from TCGA project. The patients were stratified into different prognosis subsets in a sample by determining the expression level of markers (Additional file 2: Figure S4). Survival analysis of three genes (ABLIM1, $p=0.019 ;$ ATXN1, $p=0.029$; PLEKHA1, $p=0.048)$ revealed patient stratification with statistical significance on overall survival analysis. Interestingly, we observed that ABLIM1 and ATXN1 were significantly associated with overall survival for these different prognosis prediction tools.

\section{The GMI signature-based prognosis models improve AML} survival prediction

To test whether the GMI signature can predict AML recurrence, we developed prognosis model using the identified signature as features and evaluated performance of the models in independent cohorts. We developed a RSF prognosis model with the GMI signature using TCGAPO dataset. Variable importance (VIMP) is used to measure the increase (or decrease) in prediction error for the forest ensemble when a variable is randomly "noised-up" [55]. VIMP evaluates the predictive performance of the GMI signature and a large VIMP value indicates a potentially predictive variable. As shown in Fig. 4a, SGPP1 and CDK6 are potentially predictive features with larger positive VIMP values in model development.

In the RSF prognosis model development, five-fold cross validation was used to optimize the parameters for the RSF algorithm, and a full model based on the complete dataset was developed using the optimal parameters. The best performing parameters (ntree $=10$, $m$ try $=20)$ were selected to build the RSF prognosis model. RSF-based scores were then calculated for individual sample. The calculated RSF-based scores displayed $78 \%$ concordance $(C$-index $=0.78)$ in the light of the real survival data in the training cohort. Based on the RSF-based scores, the samples were divided into a "high-risk" group with above-median scores and a "lowrisk" group with below-median scores. As shown in Fig. $4 \mathrm{~b}$, the Kaplan-Meier analyses exhibited highly significant differences in time to overall survival between two different risk groups (hazard ratio [HR], 16.4; 95\% confidence interval $[\mathrm{CI}], 5.68-47.3 ; p=1.263 \mathrm{e}-11)$. The lowrisk group had 3-year overall survival rate of $100 \%$ and the high-risk group possessed 3-year overall survival rate of $26 \%$. The developed RSF prognosis model was applied for the independent test dataset and predictive performance was evaluated using the cohort TCGAPT. As 


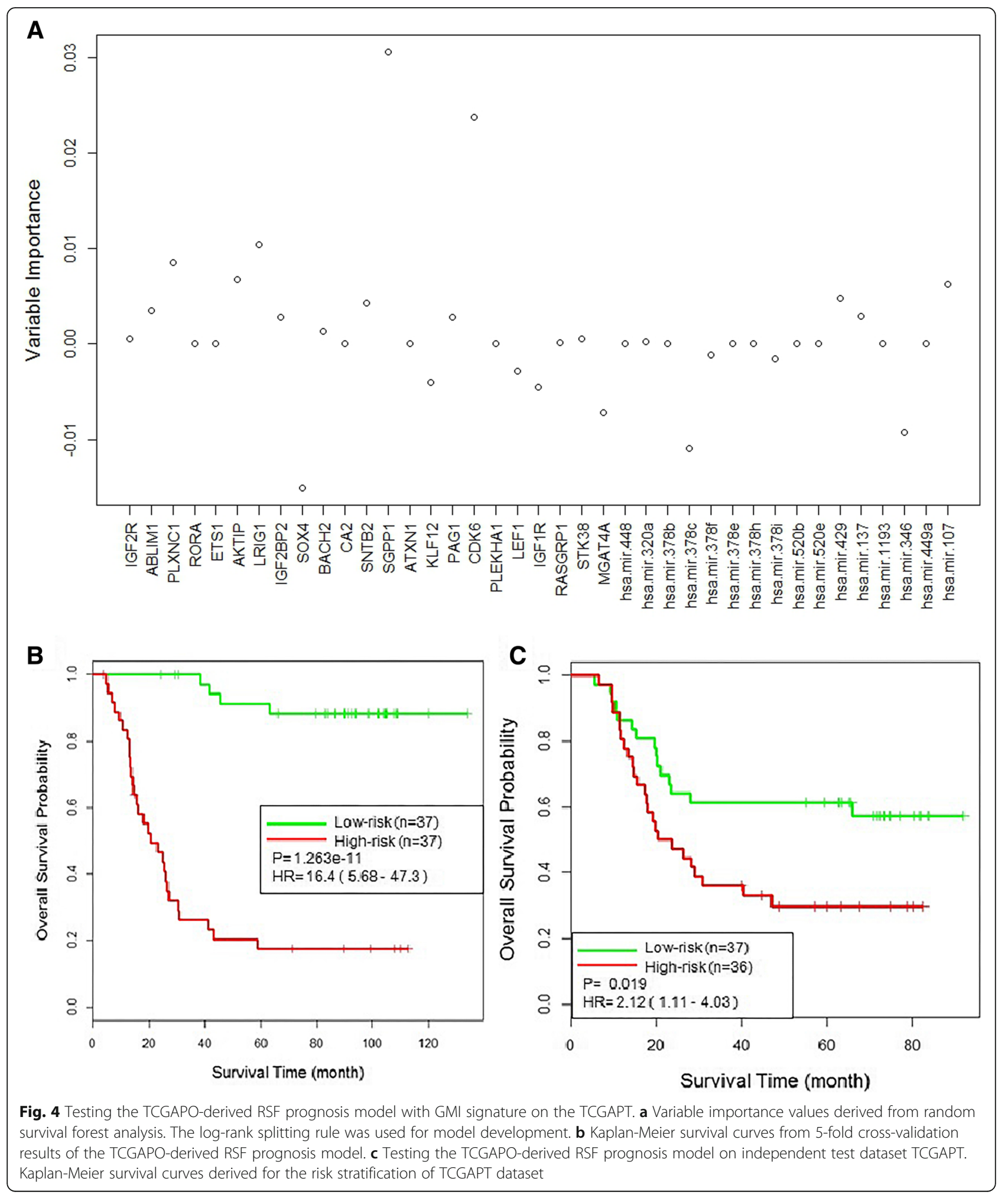

shown in Fig. 4c, the high-risk group showed significantly worse overall survival $(C$-index $=0.59$, hazard ratio $[\mathrm{HR}], 2.12$; 95\% confidence interval $[\mathrm{CI}], 1.11-4.03$; $p=0.019)$ than the low-risk group. The overall survival at 3 years between low-risk and high-risk group was 63 and $35 \%$ respectively.

To further evaluate the effectiveness of the GMI signature, we reversed the training and testing datasets by 


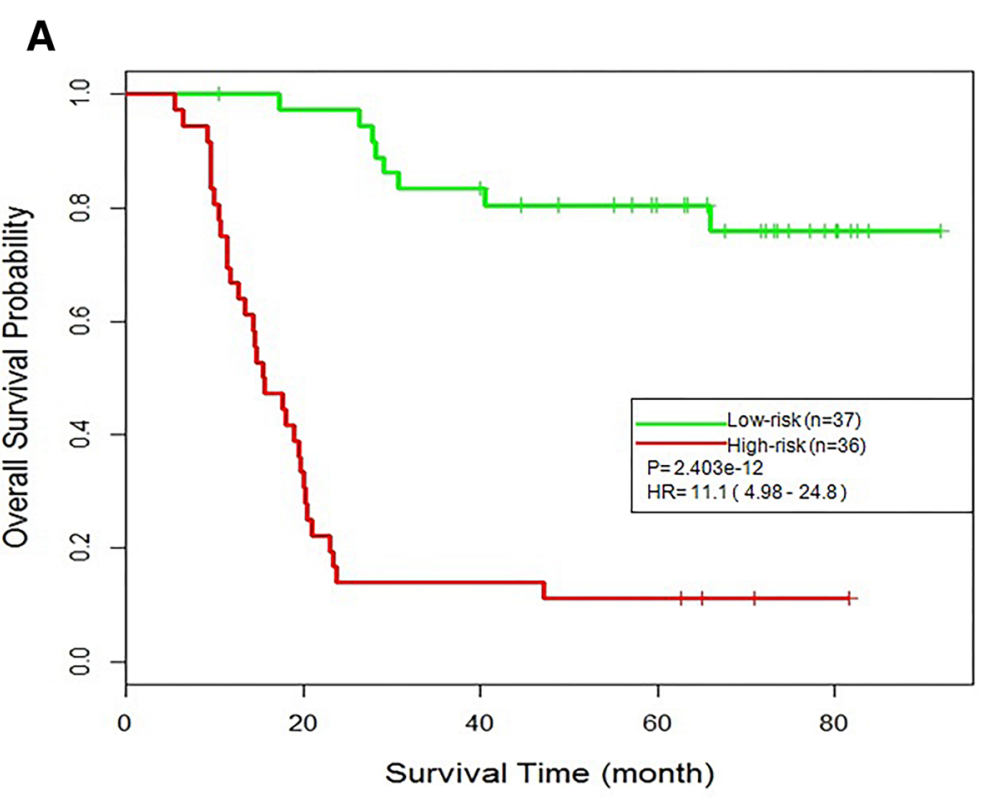

B

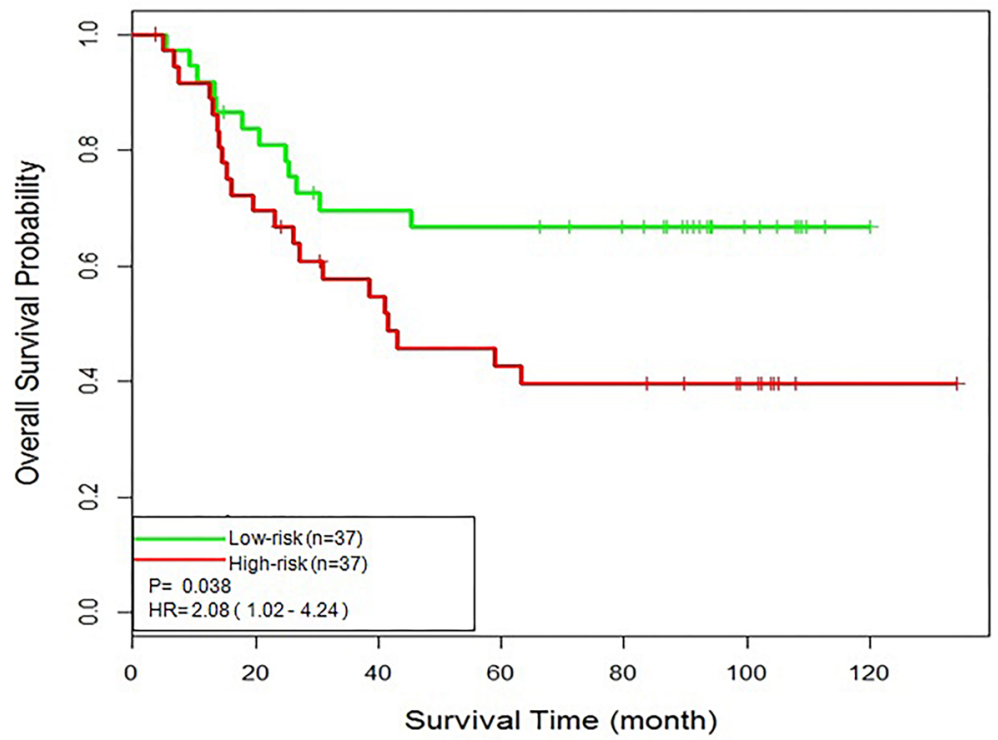

Fig. 5 Testing the TCGAPT-derived RSF prognosis model with GMI signature on the TCGAPO. a Kaplan-Meier survival curves from 5-fold crossvalidation results of the TCGAPT-derived RSF prognosis model. $\mathbf{b}$ Testing the TCGAPT-derived RSF prognosis model on independent test dataset TCGAPO. Kaplan-Meier survival curves derived for the risk stratification of TCGAPO dataset

constructing RSF prognosis model based on TCGAPT dataset and testing their performance on TCGAPO dataset. Analogous results were achieved as shown in Fig. 5. As shown in Fig. 5a, scores derived from the GMI signature-based model showed $77 \%$ concordance (Cindex $=0.77$ ) when compared to the real survival data and the patients were seperated into two groups with significantly different overall survival (hazard ratio [HR], 11.1; 95\% confidence interval [CI], 4.98-24.8; $p=2.403 \mathrm{e}-$ 12). The 3 -year overall survival rate was $81 \%$ in the lowrisk group compared with $17 \%$ in the high-risk group.
The TCGAPT-derived RSF prognosis model was test on independent test dataset TCGAPO. As shown in Fig. 5b, the Kaplan-Meier analyses for overall analysis illustrated the difference between the high-risk and low-risk group was highly significant $(C$-index $=0.58$, hazard ratio $[\mathrm{HR}], 2.08$; 95\% confidence interval $[\mathrm{CI}]$, $1.02-4.24 ; p=0.038)$. The overall survival at 3 years was $71 \%$ for the low-risk group in comparison with $57 \%$ for the high-risk group. These results confirmed that the GMI signature-based prognosis models could predict AML recurrence. 
Table 4 Univariate and multivariate Cox proportional hazard regression analyses of overall survival in TCGAPT. $M$, male; $F$, female; W, white; BA, Black or African American; GMI signature score was based on RSF model developed in TCGAPO

\begin{tabular}{lllllll}
\hline & \multicolumn{2}{l}{ Univariate } & & \multicolumn{2}{l}{ Multivariate } \\
\cline { 2 - 3 } & $p$ value & $H R(95 \% \mathrm{Cl})$ & & $p$ value & $\mathrm{HR}(95 \% \mathrm{Cl})$ \\
\hline Age & 0.25 & $1.03(0.98-1.09)$ & & 0.98 & $1.00(0.94-1.07)$ \\
Gender (M or F) & 0.62 & $1.16(0.62-2.17)$ & 0.78 & $1.09(0.58-2.06)$ \\
Race (W or BA) & 0.22 & $1.58(0.73-3.44)$ & 0.21 & $1.69(0.75-3.84)$ \\
WBC count & 0.02 & $1.79(0.91-3.95)$ & 0.03 & & $1.83(0.81-4.01)$ \\
NPM mutation & 0.35 & $1.05(0.99-1.12)$ & & 0.41 & & $0.98(0.89-1.09)$ \\
FLT3-ITD mutation & 0.016 & $2.23(1.18-4.09)$ & 0.03 & $2.08(1.04-3.95)$ \\
CEBPA mutation & 0.85 & $1.18(0.82-2.37)$ & & 0.67 & & $1.36(0.83-3.17)$ \\
WT1 mutation & 0.78 & $1.31(0.86-2.35)$ & & 0.83 & $0.87(0.68-1.59)$ \\
GMI score & 0.019 & $2.11(1.11-4.03)$ & 0.02 & $2.15(1.08-4.29)$ \\
\hline
\end{tabular}

\section{Prognostic value of the GMI signature score compared to} clinical variable

Samples of TCGAPT were analyzed using univariate and multivariate analyses with Cox's proportional hazards regression to evaluate the prognostic value of the GMI signature score in combination with individual clinical variables (age, gender, race) and risk factors (WBC count, NPM mutation, FLT3-ITD mutation, CEBPA mutation, WT1 mutation). The GMI signature-based RSF model from TCGAPO was developed to test TCGAPT and achieved the GMI signature score of the samples. Univariate and multivariate Cox's regression analyses were summarized in Table 4. The GMI signature score was significantly associated with overall survival ( $p=$ $0.019)$ in the univariate analysis and still preserved the significance $(p=0.02)$ in the multivariate analysis. As can be seen from the Table 4, there was a significant difference in overall survival with WBC count and FLT3ITD mutation $(p<0.05)$, demonstrating the potential value of two risk factors. The GMI signature score was more highly prognostic with overall survival than individual clinical variables and risk factors when the log-rank $p$ values were observed and compared. In summary, the GMI signature score represented a prognostic signature strongly associated with a higher risk of tumor recurrence.

\section{Discussion}

MicroRNAs play crucial regulatory roles in mediating mRNA degradation with a sequence-specific manner [56]. Most previous work has focused on the experimental and computational approaches to decipher how miRNAs and genes interact in cellular network [57]. The understanding of modular organization of biological network further provides a global view on the miRNAmRNA regulatory relationships. Previous studies have shown that the increased expression of $m i R-449$ causes down-regulation of oncogene CDK6 which stimulates cell proliferation in gastric cancer [58], miR-448 suppresses proliferation and invasion by regulating IGF1R in colorectal cancer cells [59], and $m i R-378$ family members target IGF1R, a key signaling molecule in rhabdomyosarcoma [60]. Based on these observations, the functional miRNA-mRNA regulatory module may be associated with cell proliferation, apoptosis and cell migration in AML. In addition, we have found that ABLIM1 and ATXN1 were significantly associated with overall survival for AML. In fact, loss of CIC or ATXN1L modulates sensitivity to MEK inhibition in $R A S$-mutant cancers [61]. It demonstrates that $S R G N$ is crucial for regulating actin cytoskeletal organization associated with cell migration for cancer metastasis [62]. Interestingly, reduced expression of $S R G N$ is accompanied by downregulation of ABLIM1, LIMA1, CFL1, RAC1, RAC2 and RHOA, concomitant with decreased cell motility [62].

The RSF prognosis models based on mRNA or miRNA expression signatures from GMI signature were developed in the cohort TCGAPO. There was a significant difference between two different risk groups with mRNA signature based RSF prognosis model (C-index $=$ 0.72 ; hazard ratio $[\mathrm{HR}], 5.3 ; 95 \%$ confidence interval $[\mathrm{CI}], 2.15-11.8 ; p=5.325 \mathrm{e}-3)$ and miRNA signature based RSF prognosis model $(\mathrm{C}$-index $=0.69$; hazard ratio [HR], 2.8; 95\% confidence interval [CI], 1.05-6.83; $p=0.006)$. The RSF prognosis model was used for the independent test dataset TCGAPT and predictive performance was measured. No significant difference between the two different risk groups was evident for mRNA signature $(\mathrm{C}$-index $=0.55$, hazard ratio $[\mathrm{HR}]$, $1.22 ; 95 \%$ confidence interval $[\mathrm{CI}], 0.98-3.05 ; p=0.05$ ) and miRNA signature $(\mathrm{C}$-index $=0.54$, hazard ratio [HR], 1.69; 95\% confidence interval [CI], 1.02-3.49; $p=$ 0.06). These results demonstrated that RSF prognosis model based on mRNA signature or miRNA signature did not result in comparable performance as that from the GMI signature. The miRNA-mRNA interactions play an important role for achieving the predictive performance.

It has previously been observed that a 17 -gene stemness score (LSC17 signature) could predict recurrence risk in AML patients [63]. The 11 genes are appeared in TCGA cohort including CPXM1, EMP1, LAPTM4B, ARHGAP22, MMRN1, ZBTB46, AKR1C3, SMIM24, CDK6, NYNRIN and SOCS2. The targeted 31 miRNAs (hsa-mir-3943,hsa-mir-761,hsa-mir-765,hsa-mir-548v, hsa-mir-5739,hsa-mir-8082,hsa-mir-8089,hsa-mir-1913, hsa-mir-4290,hsa-mir-644a,hsa-mir-6132,hsa-mir-320a, hsa-mir-8054,hsa-mir-1303,hsa-mir-4313,hsa-mir-5682, hsa-mir-4426,hsa-mir-4651,hsa-mir-4447,hsa-mir-646, hsa-mir-4326,hsa-mir-922,hsa-mir-1291,hsa-mir-3911, hsa-mir-3138,hsa-mir-1179,hsa-mir-449a,hsa-mir-4481, hsa-mir-4498,hsa-mir-4657,hsa-mir-8064) are identified 
with MiRWalk. The RSF prognosis models based on 11 genes and 31 miRNAs were developed in the cohort TCGAPO and independently tested in the cohort TCGAPT. There was a significant difference between two different risk groups in the training cohort (Cindex $=0.79$; hazard ratio $[\mathrm{HR}], 14.2 ; 95 \%$ confidence interval $[\mathrm{CI}], 4.97-41.7 ; p=2.25 \mathrm{e}-8)$. Further test revealed that the difference between the high-risk and low-risk group was significant $(\mathrm{C}$-index $=0.60$, hazard ratio [HR], 2.54; 95\% confidence interval [CI], 1.19-5.05; $p=0.03)$. Thus, 11 genes and 31 miRNAs could predict recurrence risk and inform patient prognosis in AML.

We developed Survival Support Vector Machine (SSVM) model based on the GMI signature for survival analysis in comparison with RSF method. Two parameters $\mathrm{c}$ and $\sigma$ were implemented in the SSVM model, and different parameter combinations were formed for model development from each parameter among the candidate set $\left\{10^{-4}, 10^{-3}, 10^{-2}, 10^{-1}, 10^{0}, 10^{1}, 10^{2}, 10^{3}, 10^{4}\right\}$. Five-fold cross validation was used to identify the optimized parameters according to the C-index value. In the TCGAPO dataset, the calculated SSVM scores showed 73\% concordance $(C$-index $=0.73)$ with the real survival data. The significant differences were observed in overall survival analysis between the high-risk group and low-risk group (hazard ratio $[\mathrm{HR}], 5.39$; 95\% confidence interval [CI], $1.21-24.3 ; P=0.01)$. When applying SSVM model on the validation dataset TCGAPT, scores showed $56 \%$ concordance $(\mathrm{C}$-index $=0.56)$ when compared to the real survival data. The patients were separated into two different groups with overall survival (hazard ratio [HR], 1.23; 95\% confidence interval $[\mathrm{CI}], 0.67-3.78 ; P=0.06)$. The results indicated that RSF model achieved clearly superior performance compared to SSVM model.

For the prioritized gene expression signatures, lack of concordance is a common observation in clinical trial [64, 65]. However, several AML gene expression signatures provided the relationship with patient prognosis and survival outcome [5, 7]. It suggested that different signatures may share joint biological themes that are not obvious on the individual gene level [65]. Therefore, pathway-based analysis has been made to discover biological mechanisms underpinning concordant prognosis for different gene expression signatures [66]. Whilst it has great potential, the GMI signature based prognosis model is nevertheless limited by the small scale of currently available TCGA data. Although the performance has demonstrated that the developed RSF prognosis model is effective in improving AML survival prediction, we suppose that the concomitant increase of clinical data and large scale of training samples would ameliorate the reliability of the prognosis model for cancer treatment. Ongoing large-scale cancer genome project, e.g. TCGA project, has provided multiple molecular data for clinical cancer research. The multi-omics integration reveals the association between various genomic variables and helps to discover the complex regulatory pattern toward generated heterogeneous data including mRNA expression and miRNA expression. The NanoString technology applies color-coded molecular barcodes to hybridize directly for many different types of target molecules with high sensitivity and precision. Therefore, this emerging technology could develop the GMI signature chip as AML diagnostics tool for clinical applications.

\section{Conclusion}

We used the pooled analysis of gene expression profiling data from 861 patients to identify differentially expressed gene expression signatures. We applied miRWalk approach to integrate multiple types of transcriptomic data and discover the functional miRNA-mRNA regulatory module. In the development of prognosis model, the GMI signaturebased RSF model was used to derive the prognostic risk score and accordingly stratify the patients into a high-risk and low-risk group. The results demonstrated that the RSF prognosis model measured underlying biological characteristics which are predictive of clinical outcomes and informed the treatment in AML. In conclusion, the GMI signature based RSF prognosis model can help facilitate rational design of clinical studies by patient stratification. Notwithstanding its great potential, the GMI signature score is limited by the quality of currently available data. An important future work is to validate the clinical usefulness of the GMI signature for the developed prognosis model in AML.

\section{Additional files}

Additional file 1: Development and validation of GMI signature based random survival forest prognosis model to predict clinical outcome in acute myeloid leukemia (XLSX $32 \mathrm{~kb}$ )

Additional file 2: Figure S1. Illustration of the volcano plot for identifying the differentially expressed genes. Figure S2. MiRWalk2.0 identify miRNA-mRNA interactions from an array of experimentally verified and predicted miRNA-target interaction pairs. FigureS3. KaplanMeier curve analysis of 20 expression signatures from GMI with LinkedOmics for the overall survival in AML patients. Figure S4. Kaplan-Meier curve analysis of GMI signature with GEPIA for the overall survival in AML patients. (DOCX $1029 \mathrm{~kb}$ )

\section{Abbreviations}

AML: Acute myeloid leukemia; FDR: False discovery rate; GEO: Gene expression omnibus; GEPIA: Gene expression profiling interactive analysis; GMl: Gene expression, mirna and miRNA-mRNA interactions; HR: Hazard ratio; OS: Overall survival; RMA: Robust multichip analysis; RSF: Random survival forest; SSVM: Survival support vector machine; TCGA: The cancer genome atlas; VIMP: Variable importance 


\section{Authors' contributions}

Conceived and designed the experiments: MS. Performed the experiments: MS GX. Analyzed the data: MS GX. Contributed reagents/materials/analysis tools: MS. Wrote the paper: MS. All authors read and approved the manuscript.

\section{Funding}

This work was supported by the National Natural Science Foundation of China (Grant Nos: 61572166). Publication of this article was sponsored by the National Natural Science Foundation of China (Grant Nos: 61572166). The funder had no role in the design of the study nor in the collection, analysis, and interpretation of data, nor in writing the manuscript.

\section{Availability of data and materials}

All data generated during this study are included in this article.

\section{Ethics approval and consent to participate}

Not applicable.

\section{Consent for publication}

Not applicable.

\section{Competing interests}

The authors declare that they have no competing interests.

Received: 27 February 2019 Accepted: 30 May 2019

Published online: 26 June 2019

\section{References}

1. Grimwade $D$, Walker $H$, Oliver $F$, et al. The importance of diagnostic cytogenetics on outcome in AML: analysis of 1,612 patients entered into the MRC AML 10 trial. The Medical Research Council Adult and Children's Leukaemia Working Parties. Blood. 1998;92:2322-33.

2. Preudhomme C, Sagot C, Boissel N, et al. Favorable prognostic significance of CEBPA mutations in patients with de novo acute myeloid leukemia: a study from the acute leukemia French association (ALFA). Blood. 2002;100:2717-23.

3. Tawana K, Wang J, Renneville A, et al. Disease evolution and outcomes in familial AML with germline CEBPA mutations. Blood. 2015;126:1214.

4. Fröhling S, Schlenk RF, Breitruck J, et al. Prognostic significance of activating FLT3 mutations in younger adults (16 to 60 years) with acute myeloid leukemia and normal cytogenetics: a study of the AML Study Group UIm. Blood. 2002:100:4372-80

5. Thiede C, Koch S, Creutzig E, et al. Prevalence and prognostic impact of NPM1 mutations in 1485 adult patients with acute myeloid leukemia (AML). Blood. 2006;107:4011.

6. Hyde RK, Liu PP. GATA2 mutations lead to MDS and AML. Nat Genet. 2015; 43:926-7.

7. Krauth MT, Alpermann T, Bacher U, et al. WT1 mutations are secondary events in $A M L$, show varying frequencies and impact on prognosis between genetic subgroups. Leukemia. 2015;29:660-7.

8. Döhner H, Weisdorf DJ, Bloomfield CD. Acute myeloid leukemia. N Engl J Med. 2015;373:1136-52.

9. Garzon R, Volinia S, Liu CG, et al. MicroRNA signatures associated with cytogenetics and prognosis in acute myeloid leukemia. Blood. 2008;111: 3183-9.

10. Yuan Y, Kasar S, Underbayev C, et al. MicroRNAs in Acute Myeloid Leukemia and Other Blood Disorders. Leuk Res Treatment. 2012;2012(2012-6-17): 603830

11. Forrest ARR, Kanamorikatayama M, Tomaru $Y$, et al. Induction of microRNAs, mir-155, mir-222, mir-424 and mir-503, promotes monocytic differentiation through combinatorial regulation. Leukemia. 2010;80:\$31.

12. Lim EL, Trinh DL, Ries RE, et al. MicroRNA Expression-Based Model Indicates Event-Free Survival in Pediatric Acute Myeloid Leukemia. J Clin Oncol. 2017. https://doi.org/10.1200/JCO.2017.74.7451.

13. Chuang M, Chiu YC, Chou W, et al. A 3-microRNA scoring system for prognostication in de novo acute myeloid leukemia patients. Leukemia. 2015;29:1051-9

14. Papaemmanuil E, Gerstung M, Bullinger L, et al. Genomic classification and prognosis in acute myeloid leukemia. N Engl J Med. 2016;374:2209-21.
15. Noren DP, Long BL, Norel R, et al. A crowdsourcing approach to developing and assessing prediction algorithms for AML prognosis. PLoS Comput Biol. 2016;12:e1004890

16. Kim D, Joung J-G, Sohn K-A, et al. Knowledge boosting: a graph-based integration approach with multi-omics data and genomic knowledge for cancer clinical outcome prediction. J Am Med Inform Assoc. 2015;22:109-20.

17. Russ AC, Sander S, Lück SC, et al. Integrative nucleophosmin mutationassociated microRNA and gene expression pattern analysis identifies novel microRNA - target gene interactions in acute myeloid leukemia. Haematologica. 2011;96:1783-91.

18. Chiu YC, Tsai MH, Chou WC, et al. Prognostic significance of NPM1 mutation-modulated microRNA-mRNA regulation in acute myeloid leukemia. Leukemia. 2016:30:274-84.

19. Irizarry RA, Hobbs B, Collin F, et al. Exploration, normalization, and summaries of high density oligonucleotide array probe level data. Biostatistics. 2003;4:249-64

20. Giorgi FM, Bolger AM, Lohse $M$, et al. Algorithm-driven artifacts in median polish summarization of microarray data. Bmc Bioinf. 2010;11:553.

21. Le DR, Taussig DC, Ramsay AG, et al. Peripheral blood T cells in acute myeloid leukemia (AML) patients at diagnosis have abnormal phenotype and genotype and form defective immune synapses with AML blasts. Blood. 2009;114:3909-16

22. Schneider $V$, Zhang $L$, Rojewski $M$, et al. Leukemic progenitor cells are susceptible to targeting by stimulated cytotoxic T cells against immunogenic leukemia-associated antigens. Int J Cancer. 2015;137:2083-92.

23. Ek VDH, Neumann M, Vosberg S, et al. Molecular alterations in bone marrow mesenchymal stromal cells derived from acute myeloid leukemia patients. Leukemia. 2016:31:1069.

24. Bresolin S, Zecca M, Flotho C, et al. Gene expression-based classification as an independent predictor of clinical outcome in juvenile myelomonocytic leukemia. J Clin Oncol. 2010;28:1919-27.

25. Payton JE, Grieselhuber NR, Chang LW, et al. High throughput digital quantification of mRNA abundance in primary human acute myeloid leukemia samples. J Clin Investig. 2009;119:1714-26.

26. Mougeot JLC, Bahrani-Mougeot FK, Lockhart PB, et al. Microarray analyses of oral punch biopsies from acute myeloid leukemia (AML) patients treated with chemotherapy. Oral Surg Oral Med Oral Pathol Oral Radiol Endod. 2011;112:446-52

27. Majeti R, Becker MW, Tian Q, et al. Dysregulated gene expression networks in human acute myelogenous leukemia stem cells. Proc Natl Acad Sci U S A. 2009;106:3396.

28. Krejci $\mathrm{O}$, Wunderlich $\mathrm{M}$, Geiger $\mathrm{H}$, et al. p53 signaling in response to increased DNA damage sensitizes AML1-ETO cells to stress-induced death. Blood. 2008;111:2190-9.

29. Silva FP, Swagemakers SM, Erpelinckverschueren C, et al. Gene expression profiling of minimally differentiated acute myeloid leukemia: MO is a distinct entity subdivided by RUNX1 mutation status. Blood. 2009;114:3001.

30. Zatkova A, Merk S, Wendehack M, et al. AML/MDS with 11q/MLL amplification show characteristic gene expression signature and interplay of DNA copy number changes. Genes Chromosom Cancer. 2009;48:510-20.

31. Saito Y, Nakahata S, Yamakawa N, et al. CD52 as a molecular target for immunotherapy to treat acute myeloid leukemia with high EVI1 expression. Leukemia. 2011:25:921.

32. Khaznadar Z, Boissel N, Agaugué S, et al. Defective NK cells in acute myeloid leukemia patients at diagnosis are associated with blast transcriptional signatures of immune evasion. J Immunol. 2015;195:2580.

33. Bachas C, Schuurhuis GJ, Zwaan CM, et al. Gene expression profiles associated with pediatric relapsed AML. PLoS One. 2015;10:e0121730.

34. de Jonge HJ, Valk PJ, Veeger NJ, et al. High VEGFC expression is associated with unique gene expression profiles and predicts adverse prognosis in pediatric and adult acute myeloid leukemia. Blood. 2010;116:1747.

35. Guo Z, Wang A, Zhang W, et al. PIM inhibitors target CD25-positive AML cells through concomitant suppression of STAT5 activation and degradation of MYC oncogene. Blood. 2014;124:1777-89.

36. Cheung $\mathrm{AM}$, Chow $\mathrm{HC}$, Liang $\mathrm{R}$, et al. A comparative study of bone marrow and peripheral blood CD34+ myeloblasts in acute myeloid leukaemia. $\mathrm{Br}$ J Haematol. 2009;144:484-91.

37. Leonard SM, Perry T, Woodman CB, et al. Sequential treatment with Cytarabine and Decitabine has an increased anti-leukemia effect compared to Cytarabine alone in xenograft models of childhood acute myeloid leukemia. PLoS One. 2014;9:e87475. 
38. Salvestrini $V$, Zini R, Rossi $L$, et al. Purinergic signaling inhibits human acute myeloblastic leukemia cell proliferation, migration, and engraftment in immunodeficient mice. Blood. 2012;119:217.

39. Taskesen E, Bullinger L, Corbacioglu A, et al. Prognostic impact, concurrent genetic mutations, and gene expression features of AML with CEBPA mutations in a cohort of 1182 cytogenetically normal AML patients: further evidence for CEBPA double mutant AML as a distinctive disease entity. Blood. 2011;117:2469.

40. Falini B, Macijewski K, Weiss T, et al. Multilineage dysplasia has no impact on biologic, clinicopathologic, and prognostic features of AML with mutated nucleophosmin (NPM1). Blood. 2010;115:3776.

41. Miesner M, Haferlach C, Bacher U, et al. Multilineage dysplasia (MLD) in acute myeloid leukemia (AML) correlates with MDS-related cytogenetic abnormalities and a prior history of MDS or MDS/MPN but has no independent prognostic relevance: a comparison of 408 cases classified as "AML not otherwise specified" (AML-NOS) or "AML with myelodysplasiarelated changes" (AML-MRC). Blood. 2010;116:2742-51.

42. Mora-Jensen $\mathrm{H}$, Jendholm J, Rapin $\mathrm{N}$, et al. Cellular origin of prognostic chromosomal aberrations in AML patients. Leukemia. 2015;29:1785-9.

43. Grossmann $V$, Tiacci $E$, Holmes $A B$, et al. Whole-exome sequencing identifies somatic mutations of BCOR in acute myeloid leukemia with normal karyotype. Blood. 2011;118:6153.

44. Benjamini $Y$, Hochberg Y. Controlling the false discovery rate - a practical and powerful approach to multiple testing. J R Stat Soc. 1995;57:289-300.

45. Dweep H, Gretz N. miRWalk2.0: a comprehensive atlas of microRNA-target interactions. Nat Methods. 2015;12:697.

46. Vasaikar SV, Straub P, Wang J, et al. LinkedOmics: analyzing multi-omics data within and across 32 cancer types. Nucleic Acids Res. 2017;46: D956-D963.

47. Tang Z, Li C, Kang B, et al. GEPIA: a web server for cancer and normal gene expression profiling and interactive analyses. Nucleic Acids Res. 2017;45: W98-W102.

48. Ishwaran H, Kogalur UB. Random survival forests for R. R news. 2007;7:25-31.

49. Chi SW, Zang JB, Mele A, et al. Argonaute HITS-CLIP decodes microRNAmRNA interaction maps. Nature. 2009;460:479-86.

50. Zhang $H$, Alberichjorda $M$, Amabile $G$, et al. Sox4 is a key oncogenic target in C/EBPa mutant acute myeloid leukemia. Cancer Cell. 2013;24: 575-88.

51. Coughlin JJ, Stang SL, Dower NA, et al. RasGRP1 and RasGRP3 regulate B cell proliferation by facilitating B cell receptor-Ras signaling. J Immunol. 2005; $175: 7179$

52. Weisberg E, Nonami A, Chen Z, et al. Upregulation of IGF1R by mutant RAS in leukemia and potentiation of RAS signaling inhibitors by small-molecule inhibition of IGF1R. Clin Cancer Res. 2014;20:5483-95.

53. Antony-Debré I, Steidl U. CDK6, a new target in MLL-driven leukemia. Blood. 2014;124:5-6.

54. Metzeler KH, Heilmeier B, Edmaier KE, et al. High expression of lymphoid enhancer-binding factor-1 (LEF1) is a novel favorable prognostic factor in cytogenetically normal acute myeloid leukemia. Blood. 2012;120:2118

55. Breiman L. Random forests. Mach Learn. 2001:45:5-32.

56. Bartel DP. MicroRNAs: genomics, biogenesis, mechanism, and function. cell. 2004:116:281-97

57. Cui Q, Yu Z, Purisima EO, et al. Principles of microRNA regulation of a human cellular signaling network. Mol Syst Biol. 2006;2:46.

58. Bou KT, Futomakazmierczak E, Jacobsen A, et al. miR-449 inhibits cell proliferation and is down-regulated in gastric cancer. Mol Cancer. 2011; 10:29.

59. Li B, Ge L, Li M, et al. miR-448 suppresses proliferation and invasion by regulating IGF1R in colorectal cancer cells. Am J Transl Res. 2016;8:3013.

60. Megiorni F, Cialfi S, Mcdowell HP, et al. Deep sequencing the microRNA profile in rhabdomyosarcoma reveals down-regulation of miR-378 family members. BMC Cancer. 2014;14:1-17.

61. Wang B, Krall EB, Aguirre AJ, et al. ATXN1L, CIC, and ETS transcription factors modulate sensitivity to MAPK pathway inhibition. Cell Rep. 2017; 18:1543-57

62. Bay BH, Chua PJ, Yip GW-C, et al. Serglycin regulates cytoskeletal-related proteins associated with cell motility in breast Cancer in vitro. FASEB J. 2017;31:931.933.

63. Ng SWK, Mitchell A, Kennedy JA, et al. A 17-gene stemness score for rapid determination of risk in acute leukaemia. Nature. 2016;540:433.
64. Ein-Dor L, Zuk O, Domany E. Thousands of samples are needed to generate a robust gene list for predicting outcome in cancer. Proc Natl Acad Sci U S A. 2006;103:5923-8.

65. Massague J. Sorting out breast-cancer gene signatures. N Engl J Med. 2007; 356:294-7.

66. Bild AH, Yao G, Chang JT, et al. Oncogenic pathway signatures in human cancers as a guide to targeted therapies. Nature. 2006;439:353-7.

\section{Publisher's Note}

Springer Nature remains neutral with regard to jurisdictional claims in published maps and institutional affiliations.
Ready to submit your research? Choose BMC and benefit from:

- fast, convenient online submission

- thorough peer review by experienced researchers in your field

- rapid publication on acceptance

- support for research data, including large and complex data types

- gold Open Access which fosters wider collaboration and increased citations

- maximum visibility for your research: over $100 \mathrm{M}$ website views per year

At BMC, research is always in progress.

Learn more biomedcentral.com/submissions 